\title{
Gastric pathology in patients with common variable immunodeficiency
}

\author{
A Zullo, A Romiti, V Rinaldi, A Vecchione, S Tomao, F Aiuti, L Frati, G Luzi
}

\begin{abstract}
BackgroundlAims-Common variable immunodeficiency (CVID) is an immunological disorder characterised by defective antibody production. Patients with CVID have a high risk of gastric cancer. It has been suggested that gastric cancer results from an interaction between environmental factors and a genetic predisposition. The role of Helicobacter pylori as an environmental factor in gastric carcinogenesis is of current interest. Moreover, p53 gene mutations have been reported in gastric cancer. This study focuses on the gastric pathology of patients with CVID and correlation with $H$ pylor infection.

Methods-Thirty four consecutive dyspeptic patients with CVID (mean age 49.6 years, range 14-72; 17 men) were included in the study. An upper gastrointestinal endoscopy was performed and biopsy specimens were taken from the antrum, incisura angularis, and gastric body. $\mathrm{Bi}-$ opsies were used for histological assessment, to identify the presence of $H$ pylori, and to evaluate p53 overexpression.
\end{abstract}

Results-H pylori infection was detected in 14/34 (41\%) patients. Chronic active gastritis involving both antrum and body was observed more frequently in $\mathrm{H}$ pylori positive $(79 \%)$ than $H$ pylori negative $(20 \%)$ patients $(p=0.001)$. Similarly, a histological feature of multifocal atrophic gastritis was found more frequently in infected $(50 \%)$ than uninfected patients $(10 \%)(p=$ 0.012 ). In addition, one case of gastric adenocarcinoma and another of notable dysplasia were observed in the $H$ pylori positive group. Overexpression of p53 was found in six $(18 \%)$ patients, including one with normal gastric mucosa.

Conclusions-It can be hypothesised that both $H$ pylori and $\mathbf{p} 53$ alterations play a role in the gastric carcinogenesis of patients with CVID.

(Gut 1999;45:77-81)

Keywords: common variable immunodeficiency; Helicobacter pylori; p53; pathology; carcinogenesis; gastritis

Institute of Genova

Genova, Italy

A Romiti

S Tomao

Correspondence to: Dr A Zullo, Dipartimento di Medicina Clinica-II Gastroenterologia, Viale dell’Università 37, I-00185, Roma, Italy.

Accepted for publication 2 February 1999

Common variable immunodeficiency (CVID) is a heterogeneous group of immunological disorders characterised by defective antibody production and decreased serum immunoglobulins. ${ }^{1}$ The hypogammaglobulinaemia is due to the failure of B cells to differentiate into immunoglobulin-secreting plasma cells, ${ }^{2}$ although the mechanism responsible for
B-cell differentiation failure in these patients has still not been fully clarified. ${ }^{3}$ Patients with CVID have an increased risk of contracting infection along the respiratory and gastrointestinal tracts. ${ }^{4}$ In addition, the incidence of gastric cancer in such patients is 50 -fold higher than in the general population. ${ }^{5}$

There is evidence that $H$ pylori causes chronic active gastritis, ${ }^{6}$ and a correlation has also been found between Helicobacter pylori infection and atrophy with intestinal metaplasia in the stomach. ${ }^{78}$ Indeed, recent studies have estimated a 4.5-9-fold increased risk of intestinal metaplasia in patients with this bacterial infection. ${ }^{9-11}$ Moreover, chronic atrophic gastritis with intestinal metaplasia is widely recognised as being the most prevalent precursor of intestinal type gastric carcinoma. ${ }^{12}{ }^{13}$ In addition, $H$ pylori has been classified as a group I carcinogen by the International Agency for Research on Cancer. ${ }^{14}$

Gastric cancer, like many other types of cancer, probably results from an interaction between environmental factors and a genetic predisposition. Several alterations in tumour suppressor genes (p53, APC, DCC) have been described in human gastric specimens. ${ }^{15}$ In addition, p53 point mutations have been reported not only in gastric cancer but also in precancerous lesions, suggesting that mutation of the p53 gene is an early event in gastric carcinogenesis. ${ }^{16}{ }^{17}$ Few data exist to date on the role of $H$ pylori in the gastric pathology of patients with CVID. Teahon et $a l^{18}$ found atrophic gastritis with dysplasia in one patient with CVID infected by $H$ pylori, and Shirley et $a l^{19}$ described the early appearance of atrophic gastritis in two children with agammaglobulinaemia and $H$ pylori infection. Misbah et $a l^{20}$ showed that regular administration of immunoglobulin failed to make any impact on the atrophic gastritis and $H$ pylori infection in a patient with CVID. On the other hand, no data on the overexpression of p53 in patients with CVID are available in the literature.

This study aimed to assess both histological alterations and p53 overexpression in the gastric mucosa of patients with CVID, and to correlate these findings with $H$ pylori infection.

\section{Methods}

Thirty four dyspeptic patients (mean age 49.6 years, range $14-72 ; 17$ men) out of 65 with CVID, regularly referred to our Day Hospital for Immunodeficiencies, were included in the

Abbreviations used in this paper: CVID, common variable immunodeficiency; MAG, multifocal atrophic gastritis. 
study. Dyspepsia was considered present when the patient complained of at least one of the following symptoms: discomfort in the upper abdomen, epigastric pain, heartburn, nausea/ vomiting, delayed digestion, belching. Diagnosis of CVID was made as described elsewhere. ${ }^{21}$ All patients were undergoing long term treatment (median eight years; range 1-15) with intravenous immunoglobulin (mean $150 \mathrm{mg}$ ) $\mathrm{kg}$; range 100-250) at two weekly intervals, and antibiotics, if necessary. An upper gastrointestinal endoscopy was performed and biopsy specimens were taken from the antrum, incisura angularis, and the gastric body (three specimens for each site), as recommended in the updated Sydney System classification of gastritis. ${ }^{22}$ Further biopsies were performed on gastric lesions, when present. H pylori infection was detected by the rapid urease test (CP-test; Yamanouchi, Milan, Italy) and histology (Giemsa modified staining). Patients were considered to be $H$ pylori positive if the CP-test was positive and/or bacteria were identified on histological examination. Haematoxylin and eosin staining was performed for histological assessment. Biopsy samples showing incomplete intestinal metaplasia were stained with Alcian blue, $\mathrm{pH}$ 2.5/periodic acid/Schiff reagent and high iron diamine Alcian blue, $\mathrm{pH} 2.5$, in order to identify subtypes (II or III) of intestinal metaplasia. ${ }^{23}$

Before intravenous immunoglobulin replacement, a blood sample was obtained from each patient for immunoglobulin level determination. All patients gave their informed consent to participate.

\section{IMMUNOHISTOCHEMICAL ANALYSIS}

Briefly, $5 \mu \mathrm{m}$ serial sections from paraffin wax embedded tissue were used for the immunohistochemical analysis. Paraffin wax embedded tissue sections were dewaxed in xylene and rehydrated through graded ethanols to phosphate buffered saline, $\mathrm{pH}$ 7.4. Endogenous peroxidase activity was blocked by immersion in distilled water with $0.3 \%(\mathrm{v} / \mathrm{v})$ hydrogen peroxide for 30 minutes. The specimens were then pretreated with an antigen retrieval in a microwave oven and immunostained with antip53 monoclonal antibodies (Dako, Glostrup, Denmark) for 60 minutes at room temperature in a humidified chamber. Immunohistochemistry was performed using a streptavidin-biotin system kit for primary murine antibodies (LSAB2 kit; Dako). The peroxidase reaction was initiated by the addition of $0.06 \%$ diaminobenzidine (Sigma, St Louis, MO, USA) in phosphate buffered saline containing $0.01 \%$ hydrogen peroxide. The diaminobenzidine reaction time was controlled at seven minutes. The slides were then counterstained with Harris haematoxylin and permanently mounted under a coverslip. Negative controls were performed replacing the specific antibodies with isotype-identical murine monoclonal antibodies of irrelevant specificity. The degree of immunostaining was evaluated by counting diaminobenzidine stained gastric cells out of a minimum of 500 cells in different microscopic fields at a magnification of $\times 400$ and was
Table 1 Serum immunoglobulin levels ( $\mathrm{mg} / \mathrm{dl}$ ) before a regular intravenous immunoglobulin replacement

\begin{tabular}{lll}
\hline Immunoglubulin & $\begin{array}{l}\text { H pylori positive } \\
(n=14)\end{array}$ & $\begin{array}{l}\text { H pylori negative } \\
(n=20)\end{array}$ \\
\hline IgG & $2.78(0.05)$ & $2.73(0.13)$ \\
IgA & $0.79(0.48)$ & $1(0.56)$ \\
IgM & $1.16(0.83)$ & $1.15(0.61)$ \\
\hline
\end{tabular}

Results are mean (SD) expressed as a logarithm.

Table 2 Endoscopic findings in H pylori positive and negative patients

\begin{tabular}{lll}
\hline Endoscopy finding & $\begin{array}{l}\text { H pylori } \\
\text { positive } \\
(n=14)\end{array}$ & $\begin{array}{l}\text { H pylori } \\
\text { negative } \\
(n=20)\end{array}$ \\
\hline Normal & 2 & 12 \\
Hiatal hernia and/or oesophagitis & 6 & 7 \\
Erosion & 2 & 1 \\
Duodenal ulcer & 2 & - \\
Gastric ulcer & $2^{\star}$ & - \\
\hline
\end{tabular}

^In one case histological assessment disclosed adenocarcinoma.

expressed as a percentage. The results obtained were consistent with the overall reactivity of each monoclonal antibody in every particular tissue section, as examined at low magnification. All sections were examined independently by two investigators and complete agreement was found when they were determining p53 positivity or negativity. When the positive values obtained were being observed, the interobserver variation was found to be less than $10 \%$ in each case.

STATISTICAL ANALYSIS

Data analysis was performed using Student's $t$ test for unpaired data, Fisher's exact test, and the Mann-Whitney test, as appropriate. Significance was assigned to values of $\mathrm{p}<0.05$.

\section{Results}

H pylori infection was observed in 14/34 (41\%) patients. The mean age differences between patients with and without infection were not significant, although $H$ pylori negative patients tended to be younger than $H$ pylori positive patients (48.7 (14.7) $v 51.6$ (11.1) years). In addition, there was a trend, although not statistically significant, towards lower mean serum IgA concentrations in $H$ pylori positive patients compared with those without infection (table 1). Use of antibiotics for unrelated infection (clarithromycin, amoxycillin, cefotaxime, ciprofloxacin) during the preceding two months was not different between infected and noninfected patients (4/14 $v 7 / 20$ respectively).

Table 3 Histological findings in $H$ pylori positive and negative patients

\begin{tabular}{|c|c|c|c|}
\hline Histological feature & $\begin{array}{l}\text { H pylori } \\
\text { positive } \\
(n=14)\end{array}$ & $\begin{array}{l}\text { H pylori } \\
\text { negative } \\
(n=20)\end{array}$ & $p$ Value \\
\hline Chronic diffuse active gastritis ${ }^{\star}$ & 11 & 4 & 0.001 \\
\hline Multifocal atrophic gastritist & (7) & (2) & 0.012 \\
\hline With metaplasia in the antrum & (2) & & \\
\hline With metaplasia in the body & (2) & & \\
\hline Without metaplasia & & (2) & \\
\hline Chronic antral active gastritis & 3 & 1 & \\
\hline Chronic body gastritis & & 1 & \\
\hline Normal mucosa & & 14 & $<0.001$ \\
\hline
\end{tabular}

*Intestinal metaplasia was classified as the complete type in all but one patient, who had type III incomplete metaplasia.

tOne of these patients had intestinal type adenocarcinoma and another had notable dysplasia. 


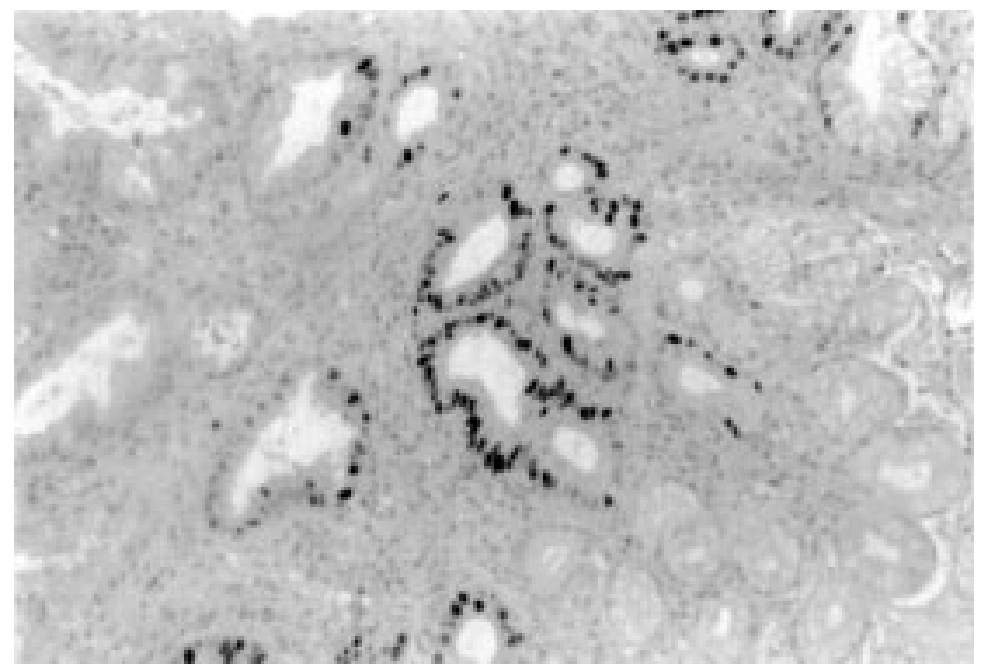

Figure 1 p53 expression in a Helicobacter pylori negative patient (14 year old girl) with normal gastric mucosa at endoscopy and with mild chronic inactive gastritis at histological assessment. Original magnification, $\times 200$.

Table 2 shows the endoscopic findings. In brief, among the $H$ pylori positive patients, two cases of duodenal ulcer and two of gastric ulcer (one with a diameter $>3 \mathrm{~cm}$ ) were observed, while among the $H$ pylori negative group endoscopy showed gastric erosions in a single patient.

Table 3 shows the histological features observed. Briefly, all $H$ pylori positive patients had chronic gastritis and 11 of these $(79 \%)$ had chronic active gastritis, involving both the antrum and body (diffuse). In seven (50\%) H pylori positive patients, there was evidence of multifocal atrophic gastritis (MAG). A case of notable dysplasia on the incisura angularis (49 year old woman) and a case of intestinal type gastric carcinoma (65 year old man) were observed in patients with MAG. Intestinal metaplasia was classified as the complete type in all but one patient ( 54 year old woman), who had type III incomplete metaplasia. Among the H pylori negative patients, four out of $20(20 \%)$ had chronic diffuse active gastritis, and two of these showed MAG. All patients had the complete type of intestinal metaplasia. Statistical analysis showed that the prevalence of chronic diffuse active gastritis was significantly higher in $H$ pylori positive patients than in those without infection ( $p=0.001)$. Moreover, MAG was observed more frequently in $H$ pylori positive than negative patients $(\mathrm{p}=0.012)$.

With regard to genetic alterations, p53 overexpression was found in six (18\%) patients. The rate of $\mathrm{p} 53$ overexpression did not differ between $H$ pylori positive and negative patients $(2 / 14 v 4 / 20 ; \mathrm{p}=0.5)$. The percentage of gastric cells displaying intense nuclear immunostaining, suggesting abnormal expression of p53, was as high as $50 \%$ in one case. No other cell types present in the tissue section were immunoreactive. Histological assessment showed normal mucosa in one case, mild chronic inactive gastritis in another, MAG in two other cases, and corpus restricted chronic atrophic gastritis in another; one patient had gastric adenocarcinoma. Figure 1 shows p53 expression in one patient.

\section{Discussion}

This study assesses histological and genetic alterations in the gastric mucosa of patients with CVID and correlates these with $H$ pylori infection. The prevalence of $H$ pylori infection was greater than that reported elsewhere. ${ }^{18} 24$ However, in these studies, the presence of the bacterium was only determined by histology (without Giemsa staining) on a single antral and a single gastric body biopsy specimen. It is possible that the prevalence of $H$ pylori infection was therefore underestimated in these studies. In addition, the general prevalence of this infection in Italy is higher than that found in the United Kingdom and United States,${ }^{25}$ increasing the likelihood of transmission to these patients. In addition, no patient was on prophylactic antibiotic therapy in our cohort.

Our results show that the prevalence of chronic active gastritis involving both antrum and body was significantly higher in $\mathrm{H}$ pylori positive $(79 \%)$ than $H$ pylori negative $(20 \%)$ patients. Moreover, MAG was observed more frequently in patients with infection $(50 \%)$ than in those without $(10 \%)$. It should also be pointed out that both of the $H$ pylori negative patients harbouring MAG had notable neutrophil and lymphocyte infiltration of the gastric mucosa, suggesting that $H$ pylori infection cannot be excluded in these two patients. ${ }^{22} 2627$ Indeed, the negative results obtained with both the rapid urease test and on histological assessment could simply depend on a low bacterial load resulting from the previous use of antibiotics (both clarithromycin six and seven weeks before) for unrelated infection.

The prevalence of MAG in $H$ pylori positive patients with CVID observed in this study is greater than that found in a recent study in Italy on 267 consecutive immunocompetent dyspeptic patients, where only $6 \%$ of the $H$ pylori positive and none of the $H$ pylori negative patients had histological features of MAG. ${ }^{11}$

It is not known why there is a higher prevalence of MAG in patients with CVID and $H$ pylori infection, although one possibility is that alterations in the production of cytokines, ${ }^{21}$ which may be involved in the gastric inflammatory cascade, ${ }^{28}$ could favour the pathogenicity of $H$ pylori in these patients. As they are prone to autoimmune disorders, ${ }^{21}$ $H$ pylori mediated autoimmunity could be involved in the pathogenesis of chronic atrophic gastritis. ${ }^{29}$ Decreased local immune responses may also be relevant, because in a previous study, we found a total absence of B cells in the gastric mucosa of patients with CVID and gastritis. ${ }^{30}$ It has also been suggested that IgA plays a major defensive role in the gastric juice, by inhibiting $H$ pylori motility and adhesion and neutralising the toxins produced. ${ }^{31}$ Although a normal antibody response does not appear to be able to eradicate H pylori ${ }^{6}$ it could be argued that the absence of IgA in patients with CVID does contribute to the pathogenicity of $H$ pylori. 
Unexpectedly, intestinal metaplasia was classified as the complete type in all but one patient. However, an increasing loss of differentiation of intestinal metaplasia over time has been suggested..$^{1132}$

Correa $^{33}$ proposed a model of human gastric carcinogenesis in which a series of pathological events, beginning with active chronic gastritis and progressing to atrophy, metaplasia, and dysplasia, can lead to cancer. In particular, MAG is associated with an increased risk of gastric carcinoma. ${ }^{34} \mathrm{H}$ pylori is frequently found along with $\mathrm{MAG}$, and its prevalence is approximately $100 \%$ in populations with a high gastric cancer risk. ${ }^{35}$ Our study showed that in patients with CVID, in whom the incidence of gastric cancer is 50-fold higher than in the general population, ${ }^{5} \mathrm{MAG}$ was significantly associated with $H$ pylori infection. Moreover, gastric adenocarcinoma, notable dysplasia, and type III intestinal metaplasia were observed in three patients with $H$ pylori infection. Thus $H$ pylori may also play a role in gastric carcinogenesis in patients with CVID.

Inactivation of p53 anti-oncogene as the result of point mutations and/or gene deletions contributes to the onset of tumours in various organs. ${ }^{36}$ The mutated protein has a longer half-life than native p53, and as such can be detected by immunohistochemical techniques. ${ }^{37}$ Nearly $50 \%$ of gastric cancers show p53 overexpression, ${ }^{16}$ and some studies report p53 gene mutations in precancerous lesions, suggesting a role in the early stages of gastric carcinogenesis. ${ }^{17}$ Nevertheless, we ${ }^{38} 39$ and others ${ }^{40}{ }^{41}$ failed to find p53 overexpression in gastric precancerous lesions, including mild to moderate dysplasia. In the present study, p53 overexpression was found in six patients, irrespective of $H$ pylori status. Histological assessment showed that one of these patients had normal mucosa, and another had only mild chronic inactive gastritis. As p53 overexpression has not previously been reported in normal gastric mucosa or gastritis, some patients with CVID, similar to patients with ataxia telangiectasia, may have impaired DNA repair mechanisms which are unrelated to $H$ pylori infection and which could predispose to cancer. ${ }^{42}$ In an experimental model it has recently been reported that p53 mutant mice have a higher proliferation index than the parent strain when infected with Helicobacter felis. ${ }^{43}$ In our study we found two patients with p53 alterations and $H$ pylori infection, and one of these patients had gastric adenocarcinoma. Follow up of these patients will be important in order to establish a role for $\mathrm{p} 53$ alterations in gastric carcinogenesis in patients with CVID.

Finally, there was no correlation between the frequency or dose of immunoglobulin replacement in those patients with and without $H$ pylori infection. Furthermore, attempts to eradicate infection with immunoglobulin replacement failed in one previously reported case. $^{20}$

In conclusion, $H$ pylori infection appears to be a risk factor for the development of MAG in patients with CVID. Moreover, p53 alterations may be a cofactor in the develop- ment of cancer in these patients. Considering the low prevalence of CVID in the general population (1:50000-200 000), ${ }^{21}$ and the high incidence of gastric cancer, ${ }^{5}$ it may be appropriate to screen for gastric abnormalities by endoscopy on a regular basis in patients with CVID.

The authors thank Professor Aldo Vecchione (Department of Experimental Medicine and Pathology, University of Rome) for kindly reviewing the manuscript and providing constructive criticism. Grateful thanks go to Simon Winn for editing the text and to Angelo Del Nero and Sandro Valia for invaluable technical assistance.

1 Report of a WHO sponsored meeting. Primary immunodeficiency diseases. Immunodeficiency Rev 1989;1:173-205.

2 Saxon A, Giorgi JV, Sherr EH, et al. Failure of B cells in common variable immunodeficiency to transit from proliferation to differentiation is associated with altered $\mathrm{B}$ cell eration to differentiation is associated with altered B cell $44-55$.

3 Spickett GP, Farrant J, North ME, et al. Common variable immunodeficiency: how many diseases? Immunol Today 1997;18:325-8.

4 Hermaszewski RA, Webster ADB. Primary hypogammaglobulinaemia: a survey of clinical manifestations and complications. $Q \mathcal{F}$ Med 1993;86:31-42.

5 Kinlen LJ, Webster ADB, Bird AG, et al. Prospective study of cancer in patients with hypogammaglobulinaemia. Lancet 1985;i:263-5.

6 Blaser MJ. Hypotheses on the pathogenesis and natural history of Helicobacter pylori-induced inflammation. Gastroenterology 1992;102:720-7.

7 Craanen ME, Dekker W, Block P, et al. Intestinal metaplasia and Helicobacter pylori: an endoscopic bioptic study of the gastric antrum. Gut 1992;33:16-20.

8 Eidt S, Stolte M. Prevalence of intestinal metaplasia in Helicobacter pylori gastritis. Scand f Gastroenterol 1994;29: 607-10.

9 Fontham ETH, Ruiz B, Perez A, et al. Determinants of Helicobacter pylori infection and chronic gastritis. Am $\mathcal{f}$ Gastroenterol 1995;90:1094-101.

10 Kuipers EJ, Uyterlinde AM, Pena AS, et al. Long-term sequelae of Helicobacter pylori gastritis. Lancet 1995;345: sequelae

11 Rugge M, Cassaro M, Leandro G, et al. Helicobacter pylori in promotion of gastric carcinogenesis. Dig Dis Sci 1996;41:950-5

12 Correa P. Precursors of gastric and esophageal cancer. Cancer 1982;50:2554-65.

13 Sipponen P, Kekki M, Haapakoski J, et al. Gastric cancer risk in chronic atrophic gastritis: statistical calculations of cross-sectional data. Int $\mathcal{f}$ Cancer 1985;35:173-7.

14 International Agency for Research on Cancer. Schistosomes, liver flukes and Helicobacter pylori. IARC Monogr Eval Carcinog Risks Hum 1994;61:177-240.

15 Uchino S, Tsuda H, Noguchi M, et al. Frequent loss of heterozygosity at the DCC locus in gastric cancer. Cancer Res 1992;52:3099-102.

16 Starzynska T, Bromley M, Gosh A, et al. Prognostic significance of p53 overexpression in gastric and colorectal carcicance of p53 overexpression in gastric

17 Shiao YH, Rugge M, Correa P, et al. p53 alteration in gastric precancerous lesions. Am f Pathol 1994;144:511-17.

18 Teahon K, Webster AD, Price AB, et al. Studies on the enteropathy associated with primary hypogammaglobulinaemia. Gut 1994;35:1244-9.

19 Shirley LR, Marsh WH. Campylobacter pylori in children with X-linked agammaglobulinaemia [abstract]. F Allergy Clin Immunol 1989;83:A287.

20 Misbah SA, Chapel HM, Johnston BJ, et al. Attempt to reverse atrophic gastritis associated with common variable immunodeficiency. F Clin Gastroenterol 1992;15:354-5.

21 Report of a WHO Scientific Group. Primary immunodeficiency diseases. Clin Exp Immunol 1995;99(suppl 1):224.

22 Dixon FM, Genta RM, Yardley JH, et al. Classification and grading of gastritis. The updated Sydney System. Am $\mathcal{F}$ grading of gastritis. The upd
Surg Pathol 1996;20:1161-81.

23 Filipe MI, Potet F, Bogomoletz WV, et al. Incomplete sulphomucin-secreting intestinal metaplasia for gastric cancer. Preliminary data from prospective study from three centres. Gut 1985;26:1319-26.

24 Washington K, Stenzel T, Buckley R, et al. Gastrointestinal pathology in patients with common variable immunodeficiency and X-linked agammaglobulinemia. Am $\mathcal{F}$ Surg Pathol 1996;20:120-52.

25 The Eurogast Study Group. An international association between Helicobacter pylori infection and gastric cancer. Lancet 1993;341:1359-62.

26 Faigel DO, Furth EE, Childs M, et al. Histological predictors of active Helicobacter pylori infection. Dig Dis Sci 1996;5:937-43.

27 den Hartog G, Jansen JBJM, van der Meer JWM, et al. Gastric abnormalities in humoral immune deficiency syndromes. Scand $\mathcal{f}$ Gastroenterol 1992;27(suppl 194):38-40.

28 Figura N. Progress in defining the inflammatory cascade. Fur $\mathcal{f}$ Gastroenterol Hepatol 1995; 7:296-302. 
29 Negrini R, Savio A, Poiesi C, et al. Antigenic mimicry between Helicobacter pylori and gastric mucosa in the pathogenesis of body atrophic gastritis. Gastroenterology 1996;111:655-65.

30 Rahimi S, Rinaldi V, Zullo A, et al. Immunophenotype characterization of gastric mucosa lymphocytes in common variable immunodeficiency gastritis [abstract]. Gut 1997; 41(suppl 3):A1 18

31 Crabtree JE. Mucosal immune responses in Helicobacter pylori infection. Dynamic Nutrition Research 1995;4:14252.

32 Filipe MI, Barbatis C, Sandey A, et al. Expression of intestinal mucin antigens in the gastric epithelium and its relationship with malignancy. Hum Pathol 1988;19:19-26.

33 Correa P. Human gastric carcinogenesis: a multistep and multifactorial process: first American cancer society award lecture on cancer epidemiology and prevention. Cancer Res 1992;52:6735-40.

34 Correa P. Chronic gastritis: a clinico-pathological classification. Am $\mathcal{f}$ Gastroenterol 1988;83:504-9.

35 Correa P, Fox J, Fontham E, et al. Helicobacter pylori and gastric carcinoma. Serum antibody prevalence in populagions with contrasting a 74.
36 Hollestein M, Sidransky D, Vogelstein B. p53 mutations in

human cancer. Science 1991;253:49-53.
37 Wynford-Thomas D. p53 in tumor pathology: can we trust immunocytochemistry? f Pathol 1991;166:329-30.

38 Romiti A, Vecchione A, Muraro R, et al. p53 accumulation in precancerous gastric conditions. Cancer Detect Prev 1996;20:446-7.

39 Romiti A, Moretti A, Vecchione A, et al. Analysis of p53 expression in precancerous and malignant gastric mucosa. Oncology Reports 1998;5:109-13.

40 Brito MJ, Williams GT, Thompson $\mathrm{H}$, et al. Expression of p53 in early T1 gastric carcinoma and precancerous adjacent mucosa. Gut 1994;35:1697-700.

41 Joypaul BV, Newman EL, Hopwood D, et al. Expression of p53 protein in normal, dysplastic and malignant gastric mucosa: an immunohistochemical study. F Pathol 1993; 170:179-83.

42 Vorechovsky I, Scott D, Haeney MR, et al. Chromosomal radiosensitivity in common variable immunodeficiency. radiosensitivity in common
Mutat Res 1993;290:255-64.

43 Fox JG, Li X, Cahill RJ, et al. Hypertrophic gastropathy in Helicobacter felis-infected wild-type C $57 \mathrm{BL} / 6$ mice and Helicobacter felis-infected wild-type C57BL/6 mice and
p53 hemizygous transgenic mice. Gastroenterology 1996; 111:155-66. 